\title{
NASA STD-4005 - The LEO Spacecraft Charging Design Standard
}

\author{
Dale C. Ferguson" \\ NASA Marshall Space Flight Center, Huntsville, AL 35812
}

\begin{abstract}
Power systems with voltages higher than about 55 volts may charge in Low Earth Orbit (LEO) enough to cause destructive arcing. The NASA STD-4005 LEO Spacecraft Charging Design Standard will help spacecraft designers prevent arcing and other deleterious effects on LEO spacecraft. The Appendices, an Information Handbook based on the popular LEO Spacecraft Charging Design Guidelines by Ferguson and Hillard, serve as a useful explanation and accompaniment to the Standard.
\end{abstract}

\section{Introduction}

$\mathrm{T}$ HE space environment in Low Earth Orbit (LEO) is significantly different from that in geosynchronous orbit (GEO), and an increasing number of LEO satellites are being flown. Many of the interactions leading to spacecraft charging in LEO are completely different from those in GEO environments. However, for lack of a NASA standards document on LEO spacecraft charging, many spacecraft manufacturers still use the 1984 Spacecraft Charging Guidelines of Purvis, Garrett, Whittlesey, and Stevens ${ }^{1}$, intended only for GEO satellites, to design LEO spacecraft. This unfortunate state of events is being changed. The new NASA STD-4005 Low Earth Orbit Spacecraft Charging Design Standard has undergone its final review process, and is slated for release this year.

\section{Scope, Purpose and Applicability of the NASA STD-4005 Standard}

The NASA STD-4005 standard and information handbook presents an overview of the current understanding of the various plasma interactions that can result when a high voltage system is operated in the Earth's ionosphere, references common design practices that have exacerbated plasma interactions in the past, and recommends standard practices to eliminate or mitigate such reactions: The purpose of the standard is to provide a design standard for high-voltage space power systems ( $>55$ volts) that operate in the plasma environment associated with LEO (altitude from 200 and $1000 \mathrm{~km}$ and latitude between -50 and +50 degrees). Such power systems, particularly solar arrays, are the proximate cause of spacecraft charging in LEO and can interact with this environment in a number of ways that are potentially destructive to themselves as well as to the platform or vehicle that has deployed them.

High voltage systems are used in space for two reasons. The first reason is to save launch weight. First of all, for the same power level, higher voltages enable use of thinner wires (lighter cabling). This is because $\mathrm{P}=\mathrm{IV}$, and $\mathrm{V}=$ $I R$, so $P=I^{2} R$ (where $P$ is power, $I$ is current, $R$ is resistance, and V is voltage). If $I$ is decreased by use of higher $V$, then thinner wires can be used with no increase in power loss due to cabling. Of course, if one uses the same cable mass, higher voltages will enable higher efficiencies, since less power will be lost to resistance in the cables. For very large power systems, the decrease in cable mass can be substantial.

Secondly, some spacecraft functions require high voltages. For example, electric propulsion uses voltages from about $300 \mathrm{~V}$ (Hall thrusters) to about $1000 \mathrm{~V}$ (ion thrusters). For low voltage power systems, conversion of substantial power to high voltages is required for these spacecraft functions to operate. The weight of the power conversion systems, Power Management and Distribution (PMAD), can be a substantial fraction of the total power system weight in these cases. It is more efficient, and can save weight, if the high voltage functions can be directly powered from a high voltage solar array, for instance. If the high voltage function is electric propulsion, we call such a system a direct-drive electric propulsion system. Because of these and other reasons for using high voltages in space, spacecraft designiers and manufacturers are using high voltages more and more. However, the use of high voltages entails risk; in particular, spacecraft charging in LEO, in contrast to that in geosynchronous earth orbit

${ }^{*}$ Physicist, Space Environment Effects Branch, Mailstop EM50, AIAA Senior Member. 
(GEO), is caused by exposed high voltages, and can lead to arcing, power drains, power disruptions, and loss of spacecraft coatings. Thus, system designers need a standard to show them how to mitigate the spacecraft charging effects of using high voltages in LEO. In addition to system designers, NASA STD-4005 should be useful to project managers, solar array designers, system engineers, etc.

The NASA STD-4005 standard is applicable to high-voltage space power systems that operate in the plasma environment associated with LEO. As was stated before, it is intended for space systems that spend the majority of their time at altitudes between 200 and $1000 \mathrm{~km}$ (usually known as LEO applications) and at latitudes between about + and - 50 degrees - that is, space systems that do not encounter GEO (geosynchronous orbit) charging conditions, that do not (often) encounter the auroral ovals of electron streams, and that do not fly through the Van Allen belts. For the extreme radiation protection that is necessary for orbits in the Van Allen belts, exterior spacecraft charging will likely be a secondary concern. However, internal charging will be very important. It is not in the purview of NASA STD-4005 to deal with internal charging.

Some of the design standards for LEO are at variance with good design practice for GEO spacecraft. If your spacecraft will fly in both LEO and GEO conditions, care must be taken to use design solutions that are applicable in both environmental regimes.

\section{The Requirements Section}

There are sections on acronyms and definitions, defining all of the specific terms used in the Standard, that take up about 9 pages. Then comes the meat of the standard. Section 4 of the NASA STD-4005 Standard and Information Handbook is the Requirements Section. Here, all of the LEO requirements are given and references are given to the Appendices, which are the Information Handbook section, based largely on the Ferguson and Hillard (2003) LEO Spacecraft Charging Design Guidelines ${ }^{2}$. The main requirement is to prevent arcing. All of the types of arcing due to spacecraft charging are listed.

Quoting from the standard,

Arcs on spacecraft in LEO must be prevented because of their potentially disastrous consequences (see appendix $C$, section C.1.2.3). The four types of arcs which shall be prevented are as follows:

a. Solar array or power system trigger arcs (see appendix $C$, section C.1.2)

b. Sustained solar array arcs (see appendix C, section C.1.2.3.1)

c. Dielectric breakdown of structure surface coatings (can also become

sustained, see appendix $C$, section C.1.2.3.1)

d. Paschen discharges (see appendix $B$, section B.2, and appendix $D$, section D.2.3).

Strategies to prevent arcing are given, and techniques are detailed to implement the strategies. Secondly, prevention of large parasitic currents to the power system is emphasized. Strategies and techniques are given. Throughout NASA STD-4005, the importance of testing is emphasized. Many of the thresholds for arcing are poorly known, and if the strategy to prevent arcing is to keep voltages lower than the appropriate threshold, testing must be used to determine those thresholds and to guarantee that flight articles do not arc. Thus, it is listed as a requirement. The requirements section of NASA STD- 4005 is about 6 pages long.

\section{Appendices: The Information Handbook}

As stated before, the Requirements Section has many references to the Appendices, the Information Handbook Section. Based on the Ferguson and Hillard (2003) Low Earth Orbit Spacecraft Charging Design Guidelines ${ }^{2}$, the Information Handbook has been updated and revised to serve as an explanatory supplement to the NASA STD-4005 standard. It has 6 main subsections, in addition to the document reference section. The main subsections are:

APPENDIX A OVERVIEW OF PLASMA INTERACTIONS, APPENDIX B ENVIRONMENTS, 
APPENDIX C PLASMA INTERACTIONS, APPENDIX D MITIGATION TECHNIQUES, APPENDIX E MODELING, and APPENDIX F TESTING.

In each section, a comprehensive description/review of the topic is given, with many references. The Information Handbook is intended as a description of the state-of-the-art in LEO spacecraft charging, and is fully 52 pages long.

NASA STD-4005, the Low Earth Orbit Spacecraft Charging Design Standard will be published and distributed in hardcopy and as a searchable electronic file. NASA STD-4005 is approximately 83 pages long, including the Appendices and References, and may be obtained through the NASA Technical Standards Program, which has the . website $h t t p: / / s t a n d a r d s . n a s a . g o v /$.

\section{References}

\footnotetext{
I' Purvis, C. K., Garrett, H. B., Whittlesey, A. C., and Stevens, N. J., 1984, Design Guidelines for Assessing and Controlling Spacecraft Charging Effects, NASA TP-02361.

${ }_{2}^{2}$ Ferguson, D. C. and Hillard, G. B., 2003, Low Earth Orbit Spacecraft Charging Design Guidelines. NASA TP-2003212287.
} 